\title{
Instrumentos para Rastreamento de Sinais Precoces do Autismo: Revisão Sistemática
}

\author{
Mariana de Miranda Seize - Pontifícia Universidade Católica do Rio de Janeiro, Rio de Janeiro, Brasil \\ Juliane Callegaro Borsa - Pontifícia Universidade Católica do Rio de Janeiro, Rio de Janeiro, Brasil
}

\begin{abstract}
Resumo
O rastreamento dos sinais precoces do autismo é crucial para um diagnóstico também precoce, viabilizando uma intervenção efetiva que possibilite a melhora no desenvolvimento e na qualidade de vida do indivíduo com autismo. O objetivo deste estudo foi identificar os instrumentos disponíveis para rastreamento dos sinais do autismo em crianças com até 36 meses de idade. Para tanto, foi conduzida uma revisão de artigos publicados entre 2004 e 2015 nas bases de dados eletrônicas SciELO, PubMed, PsycINFO e Lilacs. Foram encontrados 11 instrumentos em 34 artigos analisados, sendo que apenas um instrumento foi traduzido para o português, o que indica uma escassez desses instrumentos no Brasil. Considerando a relevância da identificação e do diagnóstico precoce do autismo para a melhoria da qualidade de vida do sujeito, acredita-se que é essencial que estudos sobre instrumentos para rastreamento precoce sejam conduzidos no país.

Palavras-chave: autismo, criança, avaliação psicológica
\end{abstract}

Instruments to Screen the Early Signs of Autism: Systematic Review

\begin{abstract}
The screening of early signs of autism is crucial for early diagnosis, allowing an effective intervention that allows an improvement in the development and quality of life of individuals with autism. The aim of this study was to identify the available instruments to screen signs of autism in children under 36 months of age. Therefore, a review of articles published between 2004 and 2015 was conducted in the electronic databases SciELO, PubMed, Lilacs and PsycINFO. 11 instruments were found in 34 articles analyzed, and only one instrument has been translated into Portuguese, indicating an absence of these instruments in Brazil. Considering the importance of early identification and diagnosis of autism for improving the quality of life of individuals with autism, it seems essential that studies of early screening instruments need to be conducted in the country.

Keywords: autism, child, psychological assessment
\end{abstract}

\section{Instrumentos para el seguimiento de las primeras señales de Autismo: Revisión Sistemática}

\section{Resumen}

Se considera fundamental el seguimiento de las primeras señales de autismo para un diagnóstico precoz, lo que permite una intervención eficaz que posibilite la mejora del desarrollo y la calidad de vida del individuo con autismo. El objetivo de este trabajo fue identificar los instrumentos disponibles para el seguimiento de las señales de autismo en niños con hasta 36 meses de edad. Por lo tanto, se realizó una revisión de los artículos publicados entre 2004 y 2015 en las bases de datos electrónicas Scielo, PubMed, Lilacs y PsycINFO. Se encontraron 11 instrumentos en 34 artículos analizados, y sólo un instrumento ha sido traducido al portugués, señalando escasez de estos instrumentos en Brasil. Considerando la importancia de la identificación y del diagnóstico precoz del autismo para mejorar la calidad de vida del individuo, se considera que es esencial que estudios sobre estos instrumentos de seguimiento de detección precoz sean realizados en el país.

Palabras clave: autismo, niño, evaluación psicológica

O autismo, também denominado Transtorno do Espectro Autista (TEA), é atualmente considerado um transtorno do neurodesenvolvimento (APA, 2013). Seu diagnóstico é realizado de maneira clínica, por meio da observação dos comportamentos da criança (Guthrie, Swineford, Nottke, \& Wetherby, 2013), de entrevista com os pais e/ou cuidadores, do levantamento de informações acerca da história do indivíduo e também do uso de instrumentos para avaliação (Matson, Beighley, \& Tyrygin, 2012). Os instrumentos para avaliação do autismo devem ser utilizados em conjunto com as observações clínicas (Matson, Nebel-Schwalm, \& Matson, 2007), levando-se em consideração os critérios diagnósticos do Manual Diagnóstico e Estatístico de
Transtornos Mentais - DSM (APA, 2013) ou da Classificação Internacional de Doenças - CID (OMS, 1993). Importante destacar que, no Brasil, o sistema classificatório oficial é a CID.

A identificação precoce dos sinais do autismo é possível (Eaves \& Ho, 2004; Lord, 1995; Matson, Wilkins, \& Gonzalez, 2008), pois muitos desses sinais podem ser notados antes dos 36 meses de idade (Mitchell, Cardy, \& Zwaigenbaum, 2011), o que é de suma importância porque oportuniza uma intervenção também precoce (Koegel, Koegel, Ashbaugh, \& Bradshaw, 2014). Por ser a primeira infância um período de máxima plasticidade cerebral (Belsky, 2010), podese otimizar o aprendizado da criança, prevenir efeitos 
secundários negativos do transtorno, melhorar as suas habilidades funcionais e qualidade de vida (Rogers \& Vismara, 2014). Estudos apontam um ganho significativo no coeficiente de inteligência verbal (QI) e também na linguagem em crianças com autismo que passaram por uma intervenção precoce (Makrygianni \& Reed, 2010; Reichow, Barton, Boyd, \& Hume, 2012; ViruésOrtega, 2010).

De acordo com a American Academy of Pediatrics, o rastreamento dos sinais do autismo deve ser realizado entre os 18-24 meses de idade por meio de instrumentos padronizados para tal finalidade (Johnson \& Myers, 2007). Caso a criança seja identificada com sinais de risco de autismo, ela deverá ser encaminhada para uma avaliação mais abrangente de modo a confirmar o diagnóstico (Bryson, Rogers, \& Fombonne, 2003). Essa confirmação é possível já no final do segundo ano de vida da criança, embora a idade média de diagnóstico seja de 5,7 anos (Shattuck et al., 2009).

Os instrumentos para rastreamento têm por finalidade identificar os sinais precoces de risco do autismo, e não de diagnosticar o transtorno (Ibañez et al., 2014). Esses instrumentos podem ser classificados segundo duas características: o formato e o nível. Quanto ao formato, pode ser escala, questionário, etc. Com relação ao nível, pode ser considerado de Nível 1 aquele cuja finalidade é identificar as crianças com sinais de risco do autismo na população geral (população não clínica), e os de Nível 2, aqueles aplicados para identificar crianças com sinais de risco do autismo entre aquelas que já apresentam quadros de problemas de desenvolvimento (população clínica) (Barton, Dumont-Mathieu, \& Fein, 2012) (Figura 1). Entre os de Nível 1, há aqueles que foram construídos para rastrear uma diversidade de transtornos do desenvolvimento e aqueles que foram desenvolvidos para rastrear especificamente o autismo (Feldman et al., 2012). São muitos os tipos de instrumentos e o uso combinado deles pode ser mais efetivo no processo de avaliação do transtorno (Ibañez et al., 2014).

Backes, Mônego, Bosa e Bandeira (2014) conduziram uma revisão sistemática acerca das propriedades psicométricas de instrumentos para avaliação do transtorno do espectro do autismo na população brasileira, indicando uma escassez de instrumentos com essa finalidade no país. O estudo aponta apenas o Modified Checklist for Autism in Toddlers - M-CHAT como o instrumento para rastreamento de sinais precoces disponível no Brasil. Considerando, portanto, esse cenário e a relevância da identificação de sinais precoces do
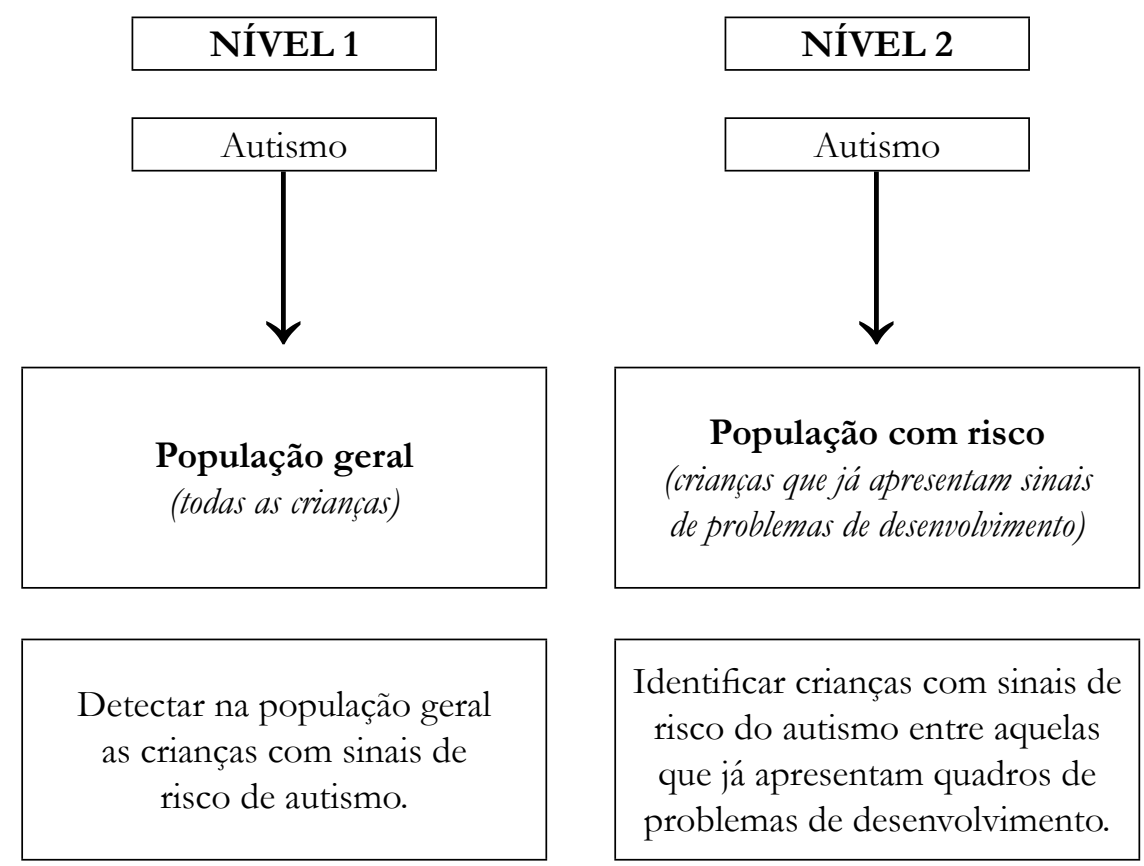

Figura 1. Nível dos instrumentos para rastreamento de sinais do autismo.

FONTE: Seize, M. M., \& Borsa, J. C. (2017). Avaliação do autismo: do rastreamento ao diagnóstico. Em M. R. C. Lins \& J. C. Borsa (Org.), Avaliação Psicológica: aspectos teóricos e práticos. Editora Vozes. 
autismo, o objetivo deste trabalho é conhecer quais são os instrumentos disponíveis para rastreamento desse transtorno utilizados em diferentes países e que possam ser aplicados em crianças com até 36 meses de idade. Espera-se com isso poder agregar evidências de pesquisas no campo da avaliação do autismo no Brasil e estimular pesquisadores da área a traduzir, adaptar e buscar evidências de validade desses instrumentos.

\section{Método}

\section{Material}

Artigos científicos com objetivo de construção, tradução, aplicação ou validação de instrumentos para rastreamento de sinais do autismo em crianças com até 36 meses de idade publicados em português, inglês ou espanhol.

\section{Procedimento}

Realizou-se a busca de artigos indexados nas bases de dados eletrônicas nos periódicos disponíveis online nas bases de dados: Scientific Electronic Library Online (SciELO), PubMed, PsycINFO e Latin American and Caribbean Health Science Literature (Lilacs).

Após a seleção das bases de dados, foram definidos os descritores. Para isso, uma busca prévia foi realizada nas bases selecionadas para confirmar a presença ou ausência de resultados para cada um dos descritores. Foram utilizados nesta revisão sistemática três eixos com os seguintes descritores para as bases de dados PubMed, PsycINFO e Lilacs: (1) "autismo" ("autism") AND (2) "ferramenta" ("tool") OR "instrumento" ("instrument") OR "checklist" ("checklist") AND (3) "identificação precoce" ("early detection") OR "primeiros sinais" ("first signs"). Para a base de dados SciELO os descritores foram: (1) "autismo" ("autism") AND (2) “ferramenta" (“tool”) OR “instrumento" (“instrument") OR “checklist" ("checklist") AND (3) "rastreamento" ("screening").

Os critérios de inclusão dos artigos foram: (a) apresentar estudos empíricos, (b) ter como população crianças com até 36 meses de idade, (c) estar publicado nos idiomas português, inglês e/ou espanhol, (d) estar disponível na íntegra na base de dados na modalidade de artigo científico e (e) ter sido publicado no período de $01 / 01 / 2004$ até 31/12/2015. Foram excluídos os artigos que apresentavam instrumentos para rastreamento de transtornos de desenvolvimento em geral, e não apenas para rastreamento específico do autismo. Também foram excluídos estudos teóricos e revisões sistemáticas. Dissertações e teses também não foram consideradas.

Inicialmente foram encontrados 499 artigos $($ SciELO $=$ três; PubMed $=271$; Lilacs $=110$; PsycINFO $=115)$. Todos esses foram exportados para um banco de dados por meio de ferramentas disponíveis nas bases de dados eletrônicas e do programa de planilhas eletrônicas Microsoft Excel. Os artigos em duplicidade foram mecanicamente eliminados $(n=$ 205), restando 294 artigos, cujos resumos foram lidos para selecionar apenas os que respeitassem os critérios de inclusão descritos anteriormente. Um total de 260 artigos foram removidos após a leitura dos resumos, restando 34 artigos finais que foram lidos na íntegra. A Figura 2 mostra um fluxograma desse processo.

\section{Análise dos Dados}

Com base nos dados finais obtidos por meio da revisão, os artigos foram categorizados de acordo com o instrumento utilizado, país, ano, periódico de publicação, área de publicação e tipo de estudo. Estabeleceu-se previamente que os artigos seriam agrupados pelos seguintes tipos de estudo: Grupo 1 - artigos de construção e/ou evidências de validade/precisão, Grupo 2 - artigos de tradução e/ou adaptação e Grupo 3 estudos de aplicação/uso do instrumento em diferentes contextos. Adicionalmente, a categorização dos instrumentos identificados nos estudos apresenta dados sobre o formato, nível, tipo de respondente e população-alvo. No caso de artigos que conduziram estudos com mais de um instrumento, foram analisados apenas os resultados daqueles específicos para rastreamento de sinais do autismo. A análise e interpretação dos dados são apresentadas nos resultados e discussão deste trabalho.

\section{Resultados e Discussão}

Foram encontrados 34 artigos, sendo 21 sobre construção e/ou evidências de validade, quatro sobre tradução e/ou adaptação e nove que apresentaram a aplicação do(s) instrumento(s) em diferentes contextos. Os Estados Unidos concentram o maior número de publicações. A Psicologia aparece como a área que mais publica. No entanto, nenhum estudo dessa área é brasileiro. Parece haver um campo de pesquisa que não está sendo explorado por parte dos psicólogos no Brasil, o que convida a uma reflexão acerca dessa lacuna. Considera-se oportuno salientar que os artigos foram em sua maioria publicados em revistas especializadas de autismo (Tabela 1). 


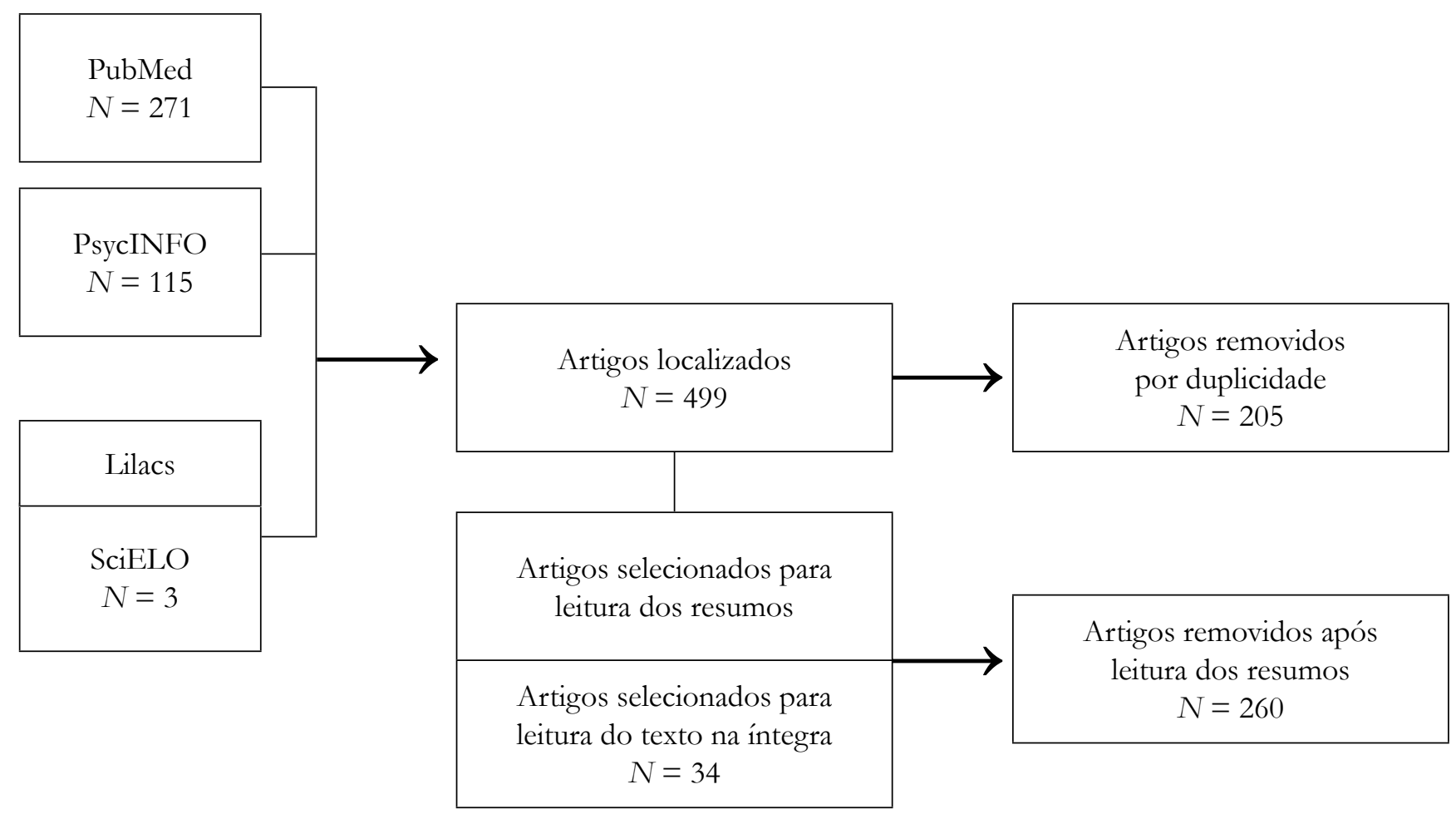

Figura 2. Fluxograma.

No total, foram identificados 11 instrumentos para rastreamento de sinais do autismo em crianças com até 36 meses de idade (Tabela 2), sendo que seis são considerados Nível 1 (para uso na população geral). Os instrumentos Nível 1 são cruciais, pois, quanto mais crianças forem rastreadas com sinais de risco na população geral e encaminhadas para uma avaliação diagnóstica, mais chances haverá de receberem um diagnóstico precocemente (Ibañez et al., 2014).

A média de idade inicial (17,72 meses) é próxima aos 18 meses recomendado pela American Academy of Pediatrics (Johnson \& Myers, 2007). No entanto, há instrumentos Nível 1 destinados ao uso em crianças com menos de 18 meses de idade. São o First Year Inventory - FYI (12 meses) e o Early Screening of Autistic Traits ESAT (14 meses) (Tabela 2).

O FYI apresentou consistência interna adequada no estudo de Reznick et al. (2007) (Tabela 3), mas um baixo valor preditivo positivo no estudo de Turner -Brown et al. (2012) (Tabela 4). Não foram encontrados dados de sensibilidade e especificidade desse instrumento. No que tange o ESAT, Swinkels et al. (2006) encontraram elevada sensibilidade (Tabela 3). Mas, os resultados obtidos por Dietz, Swinkels, van Daalen, van
Engelan, \& Buitelaar (2006) apontam para um baixo valor preditivo positivo (Tabela 4). Oosterling et al. (2009) ressaltam que o rastreamento com o ESAT possibilitou diferenciar uma criança com desenvolvimento típico de uma com desenvolvimento atípico na idade entre 8 e 44 meses. Isso indica que o ESAT talvez seja mais adequado para rastrear problemas de desenvolvimento em geral do que especificamente o autismo. Uma limitação dos instrumentos para rastreamento é apresentar elevada sensibilidade, identificando crianças que estão fora do Transtorno do Espectro Autista, embora com outros problemas de desenvolvimento e linguagem (Barbaro \& Dissanayake, 2012).

Outro instrumento Nível 1 é o Modified Checklist for Autism in Toddlers - M-CHAT, que parece ser mais adequado para detectar os sinais do autismo a partir dos 24 meses de idade (Pandey et al., 2008; Stenberg et al., 2014). Pandey et al. (2008) recomendam que, em um processo de identificação precoce, a decisão clínica deve considerar a baixa especificidade do M-CHAT no rastreamento de crianças entre 16-23 meses de idade. Segundo Stenberg et al. (2014), o rastreamento do autismo aos 18 meses de idade em uma população geral parece não ser efetivo em identificar a maioria das 
Tabela 1

Categorização Geral dos Artigos

\begin{tabular}{|c|c|c|c|c|}
\hline Autores (ano) & País & Área & Periódico & Tipo de estudo/Instrumento \\
\hline $\begin{array}{l}\text { Canal-Bedia et al. } \\
\text { (2011) }\end{array}$ & Espanha & Medicina & $\begin{array}{l}\text { Journal of Autism and } \\
\text { Developmental Disorders }\end{array}$ & $\begin{array}{l}\text { Tradução e Adaptação/ } \\
\text { M-CHAT-FUI }\end{array}$ \\
\hline Chiang et al. (2013) & Taiwan & $\begin{array}{l}\text { Psicologia, } \\
\text { Psiquiatria }\end{array}$ & $\begin{array}{l}\text { Journal of Autism and } \\
\text { Developmental Disorders }\end{array}$ & Adaptação/T-STAT \\
\hline $\begin{array}{l}\text { Choueiri e Wagner } \\
\text { (2015) }\end{array}$ & EUA & Pediatria & $\begin{array}{l}\text { The Journal of } \\
\text { Pediatrics }\end{array}$ & Construção/RITA-T \\
\hline Dietz et al. (2006) & Holanda & Psiquiatria & $\begin{array}{l}\text { Journal of Autism } \\
\text { and Developmental } \\
\text { Disorders }\end{array}$ & Evidências de validade/ESAT \\
\hline Eldin et al. (2008) & $\begin{array}{l}\text { Países } \\
\text { Árabes }\end{array}$ & $\begin{array}{l}\text { Psiquiatria, } \\
\text { Pediatria }\end{array}$ & $\begin{array}{l}\text { International Review of } \\
\text { Psychiatry }\end{array}$ & Evidências de validade/M-CHAT \\
\hline $\begin{array}{l}\text { Feldman et al. } \\
(2012)\end{array}$ & Canadá & $\begin{array}{l}\text { Psicologia, } \\
\text { Psiquiatria }\end{array}$ & $\begin{array}{l}\text { Journal of Autism and } \\
\text { Developmental Disorders }\end{array}$ & Construção/POEMS \\
\hline Hedley et al. (2010) & México & Psicologia & Autism & Evidências de validade/ADEC-SP \\
\hline Hedley et al. (2015) & EUA & Psicologia & $\begin{array}{l}\text { Journal of Autism and } \\
\text { Developmental Disorders }\end{array}$ & Evidências de validade/ADEC \\
\hline Inada et al. (2011) & Japão & $\begin{array}{l}\text { Psicologia, } \\
\text { Psiquiatria }\end{array}$ & $\begin{array}{l}\text { Research in Autism } \\
\text { Spectrum Disorders }\end{array}$ & Evidências de validade/M-CHAT \\
\hline Kara et al. (2014) & Turquia & Psiquiatria & Autism & Adaptação/M-CHAT+FUI \\
\hline $\begin{array}{l}\text { Kleinman et al. } \\
\text { (2008) }\end{array}$ & EUA & Psicologia & $\begin{array}{l}\text { Journal of Autism and } \\
\text { Developmental Disorders }\end{array}$ & $\begin{array}{l}\text { Evidências de validade/ } \\
\text { M-CHAT+FUI }\end{array}$ \\
\hline $\begin{array}{l}\text { Losapio e Pondé } \\
\text { (2008) }\end{array}$ & Brasil & Medicina & $\begin{array}{l}\text { Revista de Psiquiatria } \\
\text { do Rio Grande do } \\
\text { Sul }\end{array}$ & Tradução/M-CHAT \\
\hline Matson et al. (2010) & EUA & Psicologia & $\begin{array}{l}\text { Developmental } \\
\text { Neurohabilitation }\end{array}$ & Construção/BISCUIT-I \\
\hline $\begin{array}{l}\text { Matson, Wilkins e } \\
\text { Fodstad (2011) }\end{array}$ & EUA & Psicologia & $\begin{array}{l}\text { Journal of Autism and } \\
\text { Developmental Disorders }\end{array}$ & Evidências de validade/ BISCUIT-I \\
\hline Nah et al. (2014) & Austrália & Psicologia & Psychological Assessment & Evidências de validade/ADEC \\
\hline $\begin{array}{l}\text { Nah, Young e } \\
\text { Brewer } \\
(2014)\end{array}$ & Austrália & Psicologia & $\begin{array}{l}\text { Journal of Autism and } \\
\text { Developmental Disorders }\end{array}$ & Evidências de validade/ADEC \\
\hline Nygren et al.(2012) & Suécia & Neuropsiquiatria & $\begin{array}{l}\text { Research in Autism } \\
\text { Spectrum Disorders }\end{array}$ & Aplicação/M-CHAT \\
\hline $\begin{array}{l}\text { Oosterling et } \\
\text { al.(2009) }\end{array}$ & Holanda & Psiquiatria & $\begin{array}{l}\text { Journal of Autism and } \\
\text { Developmental Disorders }\end{array}$ & Aplicação/ESAT \\
\hline Pandey et al. (2008) & EUA & Medicina & Autism & Aplicação/M-CHAT \\
\hline $\begin{array}{l}\text { Perera, Wijewardena } \\
\text { e Aluthwela (2009) }\end{array}$ & Sri-Lanka & Medicina & $\begin{array}{l}\text { Journal of Tropical } \\
\text { Pediatrics }\end{array}$ & Aplicação/M-CHAT \\
\hline
\end{tabular}


Tabela 1

Categorização Geral dos Artigos (Continuação)

\begin{tabular}{|c|c|c|c|c|}
\hline Autores (ano) & País & Área & Periódico & Tipo de estudo/Instrumento \\
\hline Reznick et al. (2007) & EUA & $\begin{array}{l}\text { Psicologia, } \\
\text { Medicina }\end{array}$ & $\begin{array}{l}\text { Journal of Autism and } \\
\text { Developmental Disorders }\end{array}$ & Construção/FYI \\
\hline Robins et al. (2014) & EUA & Psicologia & Pediatrics & Evidências de validade/M-CHAT-R/F \\
\hline Robins (2008) & EUA & Psicologia & Autism & $\begin{array}{l}\text { Evidências de validade/ } \\
\text { M-CHAT+FUI }\end{array}$ \\
\hline Roux et al. (2012) & EUA & Medicina & $\begin{array}{l}\text { American Journal of } \\
\text { Preventive Medicine }\end{array}$ & Aplicação/M-CHAT \\
\hline $\begin{array}{l}\text { Scambler, } \\
\text { Hepburn e Rogers } \\
(2006)\end{array}$ & EUA & Psicologia & $\begin{array}{l}\text { Developmental and } \\
\text { Behaviorial Pediatrics }\end{array}$ & Aplicação/CHAT \\
\hline $\begin{array}{l}\text { Stenberg et al. } \\
\text { (2014) }\end{array}$ & Noruega & $\begin{array}{l}\text { Psicologia, } \\
\text { Psiquiaria }\end{array}$ & $\begin{array}{l}\text { Pediatric and Perinatal } \\
\text { Epidemiology }\end{array}$ & Aplicação/M-CHAT \\
\hline Stone et al. (2004) & EUA & Medicina & $\begin{array}{l}\text { Journal of Autism and } \\
\text { Developmental Disorders }\end{array}$ & Evidências de validade/STAT \\
\hline $\begin{array}{l}\text { Stone, McMahon e } \\
\text { Henderson (2008) }\end{array}$ & EUA & Medicina & Autism & Evidências de validade/STAT \\
\hline $\begin{array}{l}\text { Swinkels et al. } \\
(2006)\end{array}$ & Holanda & $\begin{array}{l}\text { Psicologia, } \\
\text { Psiquiatria }\end{array}$ & $\begin{array}{l}\text { Journal of Autism and } \\
\text { Developmental Disorders }\end{array}$ & Construção/ESAT \\
\hline $\begin{array}{l}\text { Tsai, Soong e Shyu } \\
\text { (2012) }\end{array}$ & Taiwan & Psiquiatria & Autism & $\begin{array}{l}\text { Construção/Toddler Autism Screening } \\
\text { Questionnaire }\end{array}$ \\
\hline $\begin{array}{l}\text { Turner-Brown et al. } \\
\text { (2012) }\end{array}$ & EUA & $\begin{array}{l}\text { Psicologia, } \\
\text { Psiquiatria }\end{array}$ & Autism & Evidências de validade/FYI \\
\hline $\begin{array}{l}\text { VanDenHeuvel et } \\
\text { al. (2007) }\end{array}$ & Irlanda & Medicina & Irish Medical Journal & Aplicação/CHAT \\
\hline $\begin{array}{l}\text { Wiggins, Piazza e } \\
\text { Robins (2012) }\end{array}$ & EUA & Psicologia & Autism & $\begin{array}{l}\text { Aplicação/ } \\
\text { M-CHAT+FUI }\end{array}$ \\
\hline Wong et al. (2004) & China & Medicina & Pediatrics & Construção/CHAT-23 \\
\hline
\end{tabular}

crianças que posteriormente foram diagnosticadas com o transtorno. Porém, de acordo com os autores, não se pode afirmar se a razão disso é uma ausência de sinais do transtorno aos 18 meses de idade ou se o M-CHAT não possui sensibilidade suficiente para detectar tais sinais. Vale ressaltar que a versão mais recente e revisada do instrumento, o M-CHAT-R/F (Robins et al., 2014), demanda mais estudos acerca de suas propriedades psicométricas (Tabela 4).

Entre os instrumentos de Nível 2 que podem ser utilizados antes dos 18 meses de idade, estão o Parents Observation of Early Markers Scale - POEMS (a partir de
1 mês), o Baby and Infant Screen for Children with Autism Traits - BISCUIT-I (12 meses) e o Autism Detection in Early Childhood - ADEC (Tabela 2). No entanto, os resultados apresentados por Feldman et al. (2012) indicam que o POEMS apresenta melhor sensibilidade e especificidade a partir dos 12-18 meses de idade (Tabela 3). O POEMS é o único instrumento Nível 2 baseado na observação dos pais. O BISCUIT-I, escala para aplicação por profissionais da área de saúde, apresentou consistência adequada em seus três fatores no estudo de Matson et al. (2010) (Tabela 3). Não foram encontrados dados sobre sensibilidade e especificidade dele. 
Tabela 2

Categorização dos Instrumentos

\begin{tabular}{|c|c|c|c|c|}
\hline Instrumento(s) & Formato & Nível & Tipo de respondente & População-alvo \\
\hline $\begin{array}{l}\text { (1) ADEC } \\
\text { Autism Detection in Early } \\
\text { Childhood }\end{array}$ & $\begin{array}{l}\text { Interativo } \\
\text { (16 itens) }\end{array}$ & 2 & $\begin{array}{l}\text { Psicólogos treinados em } \\
\text { aplicar o instrumento }\end{array}$ & $\begin{array}{l}\text { Crianças com 12-36 } \\
\text { meses de idade }\end{array}$ \\
\hline $\begin{array}{l}\text { (2) BISCUIT-I } \\
\text { Baby and Infant Screen for } \\
\text { Children with Autism Traits }\end{array}$ & $\begin{array}{l}\text { Escala } \\
\text { (62 itens) }\end{array}$ & 2 & $\begin{array}{l}\text { Profissionais da área de } \\
\text { saúde }\end{array}$ & $\begin{array}{l}\text { Crianças com } 12 \text { meses } \\
\text { de idade }\end{array}$ \\
\hline $\begin{array}{l}\text { (3) CHAT } \\
\text { Checklist for Autism in } \\
\text { Toddlers }\end{array}$ & $\begin{array}{l}\text { Questionário } \\
\text { (9 itens) }+ \text { Observação } \\
\text { (5 itens) }\end{array}$ & 1 & $\begin{array}{l}\text { Pais/responsáveis } \\
\text { (questionário) e } \\
\text { profissionais (observação); }\end{array}$ & $\begin{array}{l}\text { Crianças com } 18 \text { meses } \\
\text { de idade }\end{array}$ \\
\hline $\begin{array}{l}\text { (4) CHAT-23 } \\
\text { Checklist for Autism in } \\
\text { Toddlers }\end{array}$ & $\begin{array}{l}\text { Questionário } \\
\text { (23 itens) + Observação } \\
\text { (5 itens) }\end{array}$ & 1 & Pais ou responsáveis & $\begin{array}{l}\text { Crianças entre 18-24 } \\
\text { meses de idade }\end{array}$ \\
\hline $\begin{array}{l}\text { (5) ESAT } \\
\text { Early Screening of Autistic } \\
\text { Traits Questionnaire }\end{array}$ & $\begin{array}{l}\text { Questionário } \\
\text { (14 itens) }\end{array}$ & 1 & Pais/responsáveis & $\begin{array}{l}\text { Crianças entre 14-15 } \\
\text { meses de idade }\end{array}$ \\
\hline $\begin{array}{l}\text { (6) FYI } \\
\text { First Year Inventory }\end{array}$ & $\begin{array}{l}\text { Questionário } \\
\text { (63 itens) }\end{array}$ & 1 & Pais/responsáveis & $\begin{array}{l}\text { Crianças com } 12 \text { meses } \\
\text { de idade }\end{array}$ \\
\hline $\begin{array}{l}\text { (7) M-CHAT } \\
\text { Modified Checklist for } \\
\text { Autism in Toddlers }\end{array}$ & $\begin{array}{l}\text { Questionário } \\
\text { (23 itens) }\end{array}$ & 1 & $\begin{array}{l}\text { Pais/responsáveis ou } \\
\text { cuidadores }\end{array}$ & $\begin{array}{l}\text { Crianças entre 16-30 } \\
\text { meses de idade }\end{array}$ \\
\hline $\begin{array}{l}\text { (7a) M-CHAT+FUI } \\
\text { Modified Checklist for } \\
\text { Autism in Toddlers with } \\
\text { Follow-up Interview }\end{array}$ & $\begin{array}{l}\text { Questionário } \\
\text { (23 itens) + Entrevista }\end{array}$ & 1 & $\begin{array}{l}\text { Pais/responsáveis ou } \\
\text { cuidadores }\end{array}$ & $\begin{array}{l}\text { Crianças entre 16-30 } \\
\text { meses de idade }\end{array}$ \\
\hline $\begin{array}{l}\text { (7b) M-CHAT/R-F } \\
\text { Modified Checklist for } \\
\text { Autism in Toddlers, Revised } \\
\text { with Follow-up }\end{array}$ & $\begin{array}{l}\text { Questionário } \\
\text { (23 itens) + Entrevista }\end{array}$ & 1 & $\begin{array}{l}\text { Pais/responsáveis ou } \\
\text { cuidadores }\end{array}$ & $\begin{array}{l}\text { Crianças entre 16-30 } \\
\text { meses de idade }\end{array}$ \\
\hline $\begin{array}{l}\text { (8) POEMS } \\
\text { Parents Observation of } \\
\text { Early Markers Scale }\end{array}$ & $\begin{array}{l}\text { Observação } \\
\text { (61 itens) }\end{array}$ & 2 & $\begin{array}{l}\text { Pais/responsáveis ou } \\
\text { cuidadores }\end{array}$ & $\begin{array}{l}\text { Crianças entre 1-24 } \\
\text { meses de idade }\end{array}$ \\
\hline $\begin{array}{l}\text { (9) RITA-T } \\
\text { Rapid Interactive Screening } \\
\text { Test for Autism in Toddlers }\end{array}$ & $\begin{array}{l}\text { Interativo } \\
\text { (9 itens) }\end{array}$ & 2 & Profissionais de saúde & $\begin{array}{l}\text { Crianças entre 18-36 } \\
\text { meses de idade }\end{array}$ \\
\hline $\begin{array}{l}\text { (10) STAT } \\
\text { Screening Tool for Autism in } \\
\text { Toddlers \& Young Children }\end{array}$ & $\begin{array}{l}\text { Interativo } \\
\text { (12 itens) }\end{array}$ & 2 & $\begin{array}{l}\text { Profissionais com } \\
\text { experiência em autismo }\end{array}$ & $\begin{array}{l}\text { Crianças entre 24-36 } \\
\text { meses de idade }\end{array}$ \\
\hline $\begin{array}{l}\text { (11) Toddler Autism } \\
\text { Questionnaire }\end{array}$ & $\begin{array}{l}\text { Questionário } \\
\text { (15 itens) }\end{array}$ & 1 & $\begin{array}{l}\text { Pais/responsáveis ou } \\
\text { cuidadores }\end{array}$ & $\begin{array}{l}\text { Crianças entre 18-26 } \\
\text { meses de idade }\end{array}$ \\
\hline
\end{tabular}


Tabela 3

Resultados Estudos de Construção

\begin{tabular}{ll}
\hline $\begin{array}{l}\text { Instrumento } \\
\text { Artigos }\end{array}$ & Resultados \\
\hline & $\begin{array}{l}\text { População clínica: } 17-37 \text { meses }(N=1287) \\
\text { Análise fatorial exploratória identificou três fatores: } \\
\text { (1) Socialização/Comunicação Não-Verbal (23,50\% da variância); }\end{array}$ \\
BISCUIT-I & (2) Comportamento repetitivo/interesse restrito (5,66\% da variância); \\
Matson et al. & (3) Comunicação (4,06\% da variância). \\
$(2010)$ & Alfa de Cronbach FATOR $1=0,93$ \\
& Alfa de Cronbach FATOR 2 $=0,90$ \\
& Alfa de Cronbach FATOR $1=0,87$
\end{tabular}

População clinica: $18-24$ meses $(N=212)$

Questionário:

Falha em seis das 23 perguntas

CHAT-23 $\quad S_{e}=0,839 ; S p=0,848 ; \mathrm{PPV}=0,793$

Wong et al. (2004) Observação direta:

Falha em dois dos quatro itens

$S_{e}=0,736 ; S_{p}=0,912 ; \mathrm{PPV}=0,853 ;$ Inter-rater: 0,95

\section{Estudo 1:}

População geral: 8-20 meses $(N=478)$

População clínica: 87 meses $(N=153)$

ESAT

Swinkels et al. (2006)

Estudo 2:

População clínica:16-48 meses $(N=34)$

Teste-reteste: $0,81(p<0,001)$

Cutoff 3 ou mais: $S e=0,94$

FYI População geral: 12 meses $(N=1.496)$

Reznick et al. (2007) Alfa de Cronbach $=0,81$

População clínica: 1-24 meses $(N=108)$

3 meses: Alfa de Cronbach $=0,83$; Spearman Brown $=0,86 ; \mathrm{Se}=0,50 ; S p=0,87 ; \mathrm{PPV}=0,25$

6 meses: Alfa de Cronbach $=0,94 ;$ Spearman Brown $=0,95 ; \mathrm{Se}=0,25 ; \mathrm{Sp}=0,82 ; \mathrm{PPV}=0,10$

9 meses: Alfa de Cronbach $=0,90 ;$ Spearman Brown $=0,94 ; \mathrm{Se}=0,57 ; \mathrm{Sp}=0,84 ; \mathrm{PPV}=0,29$

12 meses: Alfa de Cronbach $=0,93$; Spearman Brown $=0,96 ; \mathrm{Se}=0,71 ; S p=0,68 ; \mathrm{PPV}=0,16$

18 meses: Alfa de Cronbach $=0,96 ;$ Spearman Brown $=0,99 ; \mathrm{Se}=0,89 ; \mathrm{Sp}=0,65 ; \mathrm{PPV}=0,20$

24 meses: Alfa de Cronbach $=0,97 ;$ Spearman Brown $=0,98 ; \mathrm{Se}=1,00 ; S p=0,70 ; \mathrm{PPV}=0,24$

POEMS

Feldman et al. (2012)
Teste-reteste:

2-3 meses: $r=0,93$; 3-4 meses: $r=0,91$; 4-5 meses: $r=0,48$; 5-6 meses: $r=0,92$; 7-8 meses: $r=$ 0,98; 9-10 meses: $r=0,80 ; 11-12$ meses: $r=0,82 ; 14-15$ meses: $r=0,83 ; 16-17$ meses: $r=0,94 ; 18-19$ meses: $r=0,97 ; 22-23$ meses: $r=0,98$

Validade convergente:

Domínios social e de comunicação do ASQ:

$r=-0,41$ e $r=-0,45$ respectivamente, $p$ 's $<0,01$.

Validade divergente:

Domínio movimento motor grosso do ASQ: $r=0,09$.

População clínica: 18-36 meses $(N=61)$

Correlação com ferramentas de diagnóstico específico de transtorno do espectro autista: $r=0,79 ; p<0,01$

Correlação com diagnóstico clínico de transtorno do espectro autista: $r=0,77 ; p<0,01$

Cutoff $>14: S_{e}=1.00 ; S_{p}=0,84 ; \mathrm{PPV}=0,88$

População clínica:18-26 meses $(N=77)$

Toddler Autism

Teste-reteste $-k=0,38$ a 0,92

Questionnaire

$\frac{\text { Tsai, Soong e Shyu (2012) } \quad \text { Cutoff } 5 \text { ou 6: } S_{e}=1,00 ; S_{p}=0,96 ; \mathrm{PPV}=0,90 ; \mathrm{NPV}=1,00}{\text { Nota. } S_{e}=\text { Sensibilidade; } S_{p}=\text { Especificidade; PPV }=\text { Valor Preditivo Positivo; NPV = Valor Preditivo Negativo. }}$ 
Tabela 4

Resultados Estudos de Evidências de Validade/Precisão

\begin{tabular}{ll}
\hline $\begin{array}{l}\text { Instrumento } \\
\text { Artigos }\end{array}$ & Resultados \\
\hline & População clínica: $18-36$ meses $(N=114)$ \\
& Cutoff $=11:$ Se $=0,93-0,94 ; S p=0,62-0,64$ \\
& Precisão: \\
ADEC & (a) Alfa de Cronbach $=0,80$ \\
(b) Inter-rater ICC $=0,95$ \\
Validade convergente: coeficientes de correlação entre 0,58 e 0,70 com \\
os instrumentos CARS2-ST e sub-escalas do ADOS- 2.
\end{tabular}

População clínica: $12-36$ meses $(N=215)$

Validade de critério:

Nah et al. (2014)

(a) Classificação ADOS: $k=0,66(p<0,001)$

(b) Diagnóstico do DSM-IV: $k=0,86(p<0,001)$

Precisão:

(a) Alfa de Cronbach $=0,91$

(b) Inter-rater: $r=0,98$

(c) Teste re-teste: $r=0,72$

População clínica: $19-42$ meses $(N=55)$

Nah, Young e Brewer (2014)

Validade de critério: DSM-IV-TR

Follow-up aos 2 anos: $k=0,37(p<0,01)$

Follow-up aos 6 anos: $k=0,40(p<0,05)$

Hedley et al. (2010)

População clínica: 15-73 meses $(N=115)$

$S_{e}=0,79-0,94 ; S_{p}=0,88-1,00$

População clínica: $17-37$ meses $(N=1007)$

Validade convergente:

(a) positiva com M-CHAT $(r=0,80)$;

(b) negativa com o domínio Pessoal-Social

Matson, Wilkins e Fodstad (2011)

$(r=-0,50)$ e com o domínio de comunicação $(r=-0,20)$ do BDI-2.

Validade divergente negativa com os domínios: Adaptativo $(r=-0,19)$,

Motor $(r=-0,30)$ e Cognitivo $(r=-0,44)$ do BDI2.

Precisão: Inter-rater - Kappa $=0,93, p<.001 ; 97,6 \%$

ESAT

Dietz et al. (2006)

FYI

Turner-Brown et al. (2012)

M-CHAT

Eldin et al. (2008)
População geral: $14-15$ meses $(N=31.724)$

$\mathrm{PPV}=0,25$

População geral: 12-36 meses $(N=669)$

$\mathrm{PPV}=0,31$

População clínica: $18-124$ meses $(N=228)$

$S_{e}=0,84 ; S_{p}=0,80 ; \mathrm{PPV}=0,88$ 
Tabela 4

Resultados Estudos de Evidências de Validade/Precisão (Continuação)

\begin{tabular}{|c|c|}
\hline $\begin{array}{l}\text { Instrumento } \\
\text { Artigos }\end{array}$ & Resultados \\
\hline \multirow{4}{*}{ Inada et al. (2011) } & $\begin{array}{l}\text { Inter-rater - População geral: 4-17 meses }(N=24) \\
\text { Teste-Reteste - População geral: } 4-20 \text { meses }(N=22) \\
\text { Validade de critério - População clínica: } 23-26 \text { meses }(N=25) \\
\text { Propriedades psicométricas - População geral: } 18 \text { meses }(N=1187)\end{array}$ \\
\hline & $\begin{array}{l}\text { Validade de critério: } \\
\text { CARS-TV: } r=0,581, p=0,002\end{array}$ \\
\hline & $\begin{array}{l}\text { Precisão: } \\
\text { Inter-rater: } r=0,933, p<0,001 \\
\text { Teste-reteste: } r=0,990, p<0,001\end{array}$ \\
\hline & $\begin{array}{l}2 \text { ou mais (versão longa): } S_{e}=0,75 ; S_{p}=0,893 ; \mathrm{PPV}=0,107 ; \mathrm{NPV}= \\
0,995 \\
1 \text { ou mais (versão curta): } S_{e}=0,650 ; S_{p}=0,885 ; \mathrm{PPV}=0,088 ; \mathrm{NPV}= \\
0,993\end{array}$ \\
\hline $\mathrm{M}-\mathrm{CHAT}+\mathrm{FUI}$ & $\begin{array}{l}\text { Tempo } 1 \text { - População geral: } 16-30 \text { meses }(N=3309) \text {; População Clínica: } \\
\text { 16-30 meses }(N=484) \\
\text { Tempo } 2 \text { - População Clínica: } 42-54 \text { meses }(N=1416)\end{array}$ \\
\hline Kleinman et al. (2008) & $\begin{array}{l}\text { Precisão:Alfa de Cronbach }=0,85 \\
\text { Tempo 1: PPV }=0,36 \text { (questionário); PPV }=0,74 \text { (entrevista) } \\
\text { Tempo 2: PPV = 0,38 (questionário); PPV }=0,59 \text { (entrevista) }\end{array}$ \\
\hline Robins (2008) & $\begin{array}{l}\text { População geral: } 18-24 \text { meses }(N=4.797) \\
\text { PPV }=0,57\end{array}$ \\
\hline $\begin{array}{l}\text { M-CHAT/R-F } \\
\text { Robins et al. (2014) }\end{array}$ & $\begin{array}{l}\text { População geral: } 18-24 \text { meses }(N=16.071) \\
\text { Precisão: } \\
\text { (a) Questionário: Alfa de Cronbach }=0,63 \\
\text { (b) Questionário + entrevista: Alfa de Cronbach }=0,79\end{array}$ \\
\hline $\begin{array}{l}\text { STAT } \\
\text { Stone et al. (2004) }\end{array}$ & $\begin{array}{l}\text { População clínica: } 24-35 \text { meses }(N=52) \\
\text { Cutoff } 2 \text { ou mais: } S_{e}=0,92 ; S p=0,85 ; \mathrm{PPV}=0,86 ; \mathrm{NPV}=0,92\end{array}$ \\
\hline Stone, McMahon e Henderson (2008) & $\begin{array}{l}\text { População clínica: } 12-23 \text { meses }(N=71) \\
\text { Cutoff } 2,75: \mathrm{Se}=0,95 ; S p=0,73 ; \mathrm{PPV}=0,56 ; \mathrm{NPV}=0,97\end{array}$ \\
\hline
\end{tabular}

Nota. $S_{e}=$ Sensibilidade; $S p=$ Especificidade; PPV = Valor Preditivo Positivo; NPV = Valor Preditivo Negativo.

Com relação ao ADEC, foram encontrados cinco estudos que buscam evidências de validade e precisão, indicando que esse instrumento possui propriedades psicométricas adequadas e validade convergente com o M-CHAT (Tabela 4). O ADEC mostrou ser efetivo para detectar os sinais do autismo em crianças entre 12 e 36 meses de idade (Hedley, Young, Angelica, Gallegos, \& Marcin Salazar, 2010; Nah et al., 2014). Importante 
destacar que o ADEC apresenta estrutura fatorial similar ao critério diagnóstico de Transtorno do Espectro Autista do DSM-5 (Nah et al., 2014).

O Rapid Interactive Screening Test for Autism in Toddlers - RITA-T (Choueirie \& Wagner, 2015) é um instrumento também Nível 2 que parece ser promissor para uso com crianças entre 18 e 36 meses de idade (Tabela 3). No entanto, foi encontrado apenas o estudo sobre a construção dele. Mais estudos, portanto, precisam ser conduzidos para buscar evidências de validade.

Os instrumentos Nível 1 utilizam o formato questionário, com tamanho variando entre nove-63 itens $(M=24 ; D P=17)$, indicando ser esse formato o mais adequado para uso na população geral e que possui os pais ou cuidadores como respondentes. Já entre os instrumentos Nível 2, o formato de questionário não é utilizado, preferindo-se escalas e observação. O tipo de respondente mais frequente dos instrumentos Nivel 2 são profissionais e especialistas e demandam, em geral, um maior conhecimento do autismo para serem utilizados (Tabela 2).

Apesar de o M-CHAT ser o instrumento com mais estudos $(n=14)$, não se pode afirmar que há um consenso sobre a adequação das suas propriedades psicométricas. Alguns estudos de evidências de validade apontaram para resultados inadequados (Inada et al., 2011; Kleinman et al., 2008; Robins, 2008), o mesmo ocorrendo em estudos de aplicação dele (Oosterling et al., 2009; Pandey et al., 2008; Perera, Wijewardena, \& Aluthwelage, 2009; Stenberg et al., 2014). Perera et al. (2009) consideram que uma das razões para a baixa sensibilidade $(0,25)$ do M-CHAT encontrada no Sri-Lanka talvez seja a diferença cultural e social. Por isso, sugerem que um novo instrumento seja desenvolvido. Por ter sido construído nos Estados Unidos, o M-CHAT pode não ser compreensível para a população-alvo em outros países. Entende-se que a tradução de um instrumento não garante a validade de construto, tampouco a confiabilidade da medida. É preciso considerar a pertinência dos conceitos e domínios e a adequação de cada item do instrumento para o contexto em que está sendo adaptado (Borsa, Damásio, \& Bandeira, 2012).

A eficácia do M-CHAT parece melhorar quando a entrevista follow-up anexa ao instrumento é utilizada (Kleinman et al., 2008; Roux et al., 2012). No entanto, ela não foi utilizada em seis dos estudos de aplicação encontrados (Nygren et al., 2012; Pandey et al., 2008; Perera et al., 2009; Roux et al., 2012; Stenbeg et al., 2014; Wiggins, Piazza, \& Robins, 2014). Uma das razões apontadas é a complexidade para uso em população ampla (Stenberg et al., 2014). Isso pode representar um importante desafio de uso de um instrumento cuja finalidade é rastrear os sinais precoces do autismo na população geral.

No Brasil, o Ministério da Saúde publicou as Diretrizes de Atenção à Reabilitação da Pessoa com Transtornos do Espectro do Autismo (TEA) (2014) onde insere o M-CHAT como instrumento de rastreamento adaptado e validado para uso livre no país. As referências para tal afirmação no documento fazem menção aos trabalhos de Losapio e Pondé (2008) e de Castro-Souza (2011). O estudo de Losapio e Pondé (2008) refere-se a tradução apenas dos itens do questionário M-CHAT e a dissertação de mestrado de Castro-Souza (2011) busca evidências de validade para amostra brasileira (consistência interna, sensibilidade, especificidade, valores preditivo positivo e negativo). Os resultados obtidos por Castro-Souza (2011) foram adequados mas o autor sugere a replicação do estudo.

Os estudos de Losapio e Pondé (2008) e de Castro-Souza (2011) representam um avanço, mas acredita-se que não são suficientes para considerar o instrumento validado para uso livre no país conforme indica o Ministério da Saúde (2014). Em um processo de adaptação é de suma importância comprovar as evidências psicométricas dessa nova versão e, para isso, análises estatísticas devem ser conduzidas para avaliar se o instrumento apresenta adequadas evidências de validade para o contexto em que foi adaptado (Borsa et al., 2012). No estudo de Canal-Bedia et al. (2011) adaptações culturais no M-CHAT foram necessárias e no de Kara et al. (2014) o resultado obtido foi considerado mais preciso quando o questionário foi preenchido por profissionais de saúde a partir da entrevista conduzida com os pais. Indaga-se se o mesmo poderia ocorrer no Brasil e, por isso, considera-se oportuno avaliar esta possibilidade. Além disso, a entrevista follow-up anexa ao M-CHAT, que parece melhorar a eficácia do instrumento, não foi traduzida para o português no estudo de Losapio e Pondé (2008) e não foi contemplada no de Castro-Souza (2011).

As limitações do M-CHAT precisam ser consideradas ao utilizá-lo no processo de avaliação. Por isso, acredita-se que não se deva prescindir de um clínico experiente que tenha amplo conhecimento sobre o autismo para que possa tomar a decisão mais adequada. Uma maneira de melhorar os resultados obtidos com M-CHAT talvez seja utilizá-lo como parte de um processo de avaliação mais amplo e não isoladamente. Isso corrobora outras literaturas que afirmam ser a 
aplicação de um instrumento para rastreamento apenas o início de uma etapa de avaliação (Daniels, Halladay, Shih, Elder, \& Dawson, 2014) e que o uso combinado de instrumentos pode ser mais eficaz (Ibañez et al., 2014). Os resultados obtidos por Nygren et al. (2012) apontam nessa direção, ao indicar que o resultado do M-CHAT melhora quando combinado com o uso do Joint Attention Observation (JA-OBS), um instrumento de observação composto por cinco itens para ser aplicado por enfermeiros.

Os instrumentos que apresentaram evidências de validade baseadas na relação com outras variáveis utilizando critérios diagnósticos, basearam-se no DSM-IV e DSM-IV-TR (Nah, Young \& Brewer, 2014; Nah et al., 2014). É pertinente que outros estudos sejam conduzidos para buscar novas evidências de validade em relação aos critérios diagnósticos apresentados pelo DSM-5, a mais recente versão do Manual de Estatística e Diagnóstico de Transtornos Mentais (APA, 2013). Parece relevante verificar se as mudanças nos critérios diagnósticos afetarão a avaliação e identificação dos sinais do autismo. Cabe ainda dizer que apenas estudos sobre o ADEC (Hedley et al., 2015, Matson, Wilkins, \& Fodstad, 2011; Nah et al., 2014) e o M-CHAT (Inada et al., 2011) apresentaram dados sobre convergência e/ou divergência com outros instrumentos (Tabela 4).

\section{Considerações Finais}

O presente estudo buscou identificar por meio de publicações científicas os instrumentos disponíveis para rastreamento de sinais do autismo antes dos 36 meses de idade. Considera-se oportuno ressaltar que uma limitação deste trabalho é que os artigos foram selecionados a partir de critérios pré-estabelecidos, tais como a escolha das fontes de dados e a eleição das palavras-chave para a busca. A inclusão de livros, dissertações e teses, assim como a eleição de outras palavras-chave poderia ampliar o escopo dos resultados. Isso significa que alguns instrumentos podem não ter sido contemplados.

Foram encontrados nos parâmetros desta revisão 11 instrumentos, mas não há na literatura nenhum instrumento para rastreamento considerado padrãoouro. O M-CHAT, apesar de reunir o maior número de estudos que indicam propriedades psicométricas adequadas, apresenta limitações. O ADEC é outro instrumento que é promissor mas que demanda mais estudos. Importante destacar que o M-CHAT é o único instrumento que foi traduzido para o português. No entanto, a entrevista follow-up anexa ao instrumento, que parece melhorar os resultados, não foi traduzida. Apesar de o instrumento ser recomendado pelo Ministério da Saúde (2014), diante dos resultados obtidos por meio desta revisão, acredita-se que é necessário que estudos de evidências de validade sejam conduzidos de maneira a assegurar que ele é adequado para uso no contexto brasileiro.

A escassez de instrumentos para rastreamento de sinais precoces do autismo no Brasil encontrada neste estudo de revisão corrobora com os achados de Backes et al. (2014). Isso é preocupante, pois, com isso, muitas crianças permanecem sendo diagnosticadas tardiamente sem a chance de participar de um programa de intervenção precoce que oportunize uma melhora do seu quadro clínico. Recomenda-se, portanto, que estudos sejam conduzidos de maneira a mudar esse cenário. E parece que a Psicologia tem muito a contribuir, considerando o elevado número de publicações nesta área em outros países.

\section{Referências}

American Psychiatric Association. (2013). Diagnostic and Statistical Manual of Mental Disorders (5a. ed.). Arlington, VA: American Psychiatric Publishing.

Backes, B., Mônego, B. G., Bosa, C.A., \& Bandeira, D.R. (2014). Propriedades psicométricas de instrumentos de avaliação do transtorno do espectro do autismo: Uma revisão sistemática de estudos brasileiros. Jornal Brasileiro de Psiquiatria, 63(2), 154-164.

Barbaro, J., \& Dissanayake, C. (2012). Early markers of autism spectrum disorders in infants and toddlers prospectively identified in the Social Attention and Communication Study. Autism, 17(1), 64-86.

Barton, M. L, Dumont-Mathieu, T., \& Fein, D. (2012). Screening young children for autism spectrum disorders in primary practice. Journal of Autism and Developmental Disorders., 42(6), 1165-1174.

Belsky, J. (2010). Desenvolvimento Humano: Experenciando o ciclo da vida. Tradução: Bueno D. Porto Alegre, Artmed.

Borsa, J. C., Damásio, B. F., \& Bandeira, D. R. (2012). Adaptação e validação de instrumentos psicológicos entre culturas: Algumas considerações. Paidéia, 22(53), 423-432.

Bryson, S. E., Rogers, S. J., \& Fombonne, E. (2003). Autism spectrum disorders: Early detection, Psico-USF, Bragança Paulista, v. 22, n. 1, p. 161-176, jan./abr. 2017 
intervention, education, and psychopharmacological management. Canadian Journal of Psychiatry, 48(8), 506-516.

Canal-Bedia, R., García-Primo, P., Martín-Cilleros, M. V., Santos-Borbujo, J., Guisuraga-Fernández, Z., Herráez-García, L., Herráez-García, M., M., Boada-Muñoz, L., Fuentes-Biggi, J., \& Posada-de la Paz, M. (2011). Modified checklist for autism in toddlers: Cross-cultural adaptation and validation in spain. Journal of Autism and Developmental Disorders, 41(10), 1342-1351.

Castro-Souza, R. M. (2011). Adaptação Brasileira do MCHAT (Modified Checklist for Autism in Toddlers) (Dissertação de mestrado). Universidade de Brasília, Brasília, DF, Brasil.

Chiang, C. H., Wu, C. C., Hou, Y. M., Chu, C. L., Liu, J. H., \& Soong, W. T. (2013). Development of T-STAT for early autism screening. Journal of Autism and Developmental Disorders, 43(5), 1028-1037.

Choueiri, R., \& Wagner, S. (2015). A new interactive screening test for autism spectrum disorders in toddlers. Journal of Pediatrics, 167(2), 460-466.

Daniels, A., Halladay, A. K., Shih, A., Elder, L. M., \& Dawson, G. (2014). Approachs of enhancing the early detection of autism spectrum disorders: A systematic review of literature. Journal of American Academy Child and Adolescent Psychiatry, 53(2), 141-152.

Dietz, C., Swinkels, S., van Daalen, E., van Engeland, H., \& Buitelaar, J. K. (2006). Screening for autistic spectrum disorder in children aged 14 to 15 months. II: population screening with the Early Screening of Autistic Traits Questionnaire (ESAT). Design and general findings. Journal of Autism and Developmental Disorders, 36(6), 713-722.

Eaves, L. C., \& Ho, H. H. (1996). Brief report: Stability and change in cognitive and behavioral characteristics of autism through childhood. Journal of Autism and Developmental Disorders, 26(5), 557-569.

Eldin, A. S., Habib, D., Noufal, A., Farrag, S., Bazaid, K., Al-Shabarti, M., Badr, H., Moussa, S., Essali, A., \& Gaddour, N. (2008). Use of M-CHAT for a multinational screening of young children with autism in the Arab countries. International Review of Psychiatry, 20(3), 281-289.
Feldman, M. A., Ward, R. A., Savona, D., Regehr, K., Parker, K., Hudson, Penning, H., \& Holden, J. J. (2012). Development and initial validation of a parent report measure of the behaviorial development of infants at risk for autism spectrum disorders. Journal of Autism and Developmental Disorders, 42(1), 13-22.

Guthrie, W., Swineford, L. B., Nottke, C., \& Wetherby, A. M. (2013). Early diagnosis of autism spectrum disorders: Stability and change in clinical diagnosis and symptom presentation. Journal of Child Psychology and Psychiatry, 54(5), 582-590.

Hedley, D., Nevill, R. E., Monroy-Moreno, Y., Fields, N., Wilkins, J., Butter, E., \& Mulick, J. A. (2015). Efficacy of the ADEC in Identifying Autism Spectrum Disorder in Clinically Referred Toddlers in the US. Journal of Autism and Developmental Disorders, 45, 2337-2348.

Hedley, D., Young, R., Angelica, M., Gallegos, J., \& Marcin Salazar, C. (2010). Cross-cultural evaluation of the Autism Detection in Early Childhood (ADEC) in Mexico. Autism, 14(2), 93-112.

Ibañez, L. V., Stone, W. L., \& Coonrod, E. E. (2014). Screening for Autism in Young Children. Em F. R., Volkmar, S. J., Rogers, R., Paul \& K. A. Pelphrey (Eds.), Handbook of Autism Pervasive Developmental Disorders: Assessment, interventions, and policy (4a ed.,Vol.2, pp. 585-608). Hoboken, New Jersey/ EUA: Wiley.

Inada, N., Koyama, T., Inokuchi, E., Kuroda, M., \& Kamio, Y. (2011). Reliability and validity of the Japanese version of the Modified Checklist for autism in toddlers (M-CHAT). Research in Autism Spectrum Disorders, 5(1), 330-336.

Johnson, C. P., \& Myers, S. M. (2007). American Academy of Pediatrics Council on Children With Disabilities. Identification and evaluation of children with autism spectrum disorders. Pediatrics, 120(5), 1183-1215.

Kanner, L. (1943). Autistic disturbances of affective contact. Nervous Child, 2, 217-250.

Kaufmann, P. (1996). Dicionário enciclopédico de psicanálise. Rio de Janeiro: Zahar.

Kara, B., Mukaddes, N. M., Altinkaya, I., Güntepe, D., Gökçay, G., \& Özmen, M. (2014). Using the modified checklist for autism in toddlers in a well-child 
clinic in turkey: Adapting the screening method based on culture and setting. Autism, 18(3), 331-338.

Kleinman, J. M., Robins, D. L., Ventola, P. E., Pandey, J., Boorstein, H. C., Esser, E. L., Wilson, L. B., Rosenthal, M. A., Sutera, S., Verbalis, A. D., Barton, M., Hodgson, S., Green, J., Dumont-Mathieu, T., Volkmar, F., Chawarska, K., Klin, A., \& Fein, D. (2008). The modified checklist for autism in toddlers: A follow-up study investigating the early detection of autism spectrum disorders. Journal of Autism and Developmental Disorders, 38(5), 827-839.

Koegel, L. K., Koegel, R. L., Ashbaugh, K., \& Bradshaw, J. (2014). The importance of early identification and intervention for children with or at risk for autism spectrum disorders. International Journal of Speech-Language pathology, 16(1), 50-56.

Lord, C. (1995). Follow-up of two-year-olds referred for possible autism. Journal of Child Psychology and Psychiatry, 36(8), 1365-1382.

Losapio, M. F., \& Pondé, M. P. (2008). Tradução para o português da escala M-CHAT para rastreamento precoce de autismo. Revista de Psiquiatria do Rio Grande do Sul, 30(3), 221-229.

Makrygianni, M. K., \& Reed, P. (2010) A meta-analytic review of the effectiveness of behavioural early intervention programs for children with autistic spectrum disorders. Research in Autism Spectrum Disorders, 4(4), 577-593.

Matson, J. L., Beighley, J., \& Turygin, N. (2012). Autism diagnosis and screening: Factors to consider in differential diagnosis. Research in Autism Spectrum Disorders, 6(1), 19-24.

Matson, J. L., Boisjoli, J. A., Hess, J. A., \& Wilkins, J. (2010). Factor structure and diagnostic fidelity of the Baby and Infant Screen for Children with Autism Traits-Part 1 (BISCUIT - Part 1). Developmental Neurorehabilitation, 13(2), 72-79.

Matson, J. L., Nebel-Schwalm, M., \& Matson, M. L. (2007). A review of methodological issues in the differential diagnosis of autism spectrum disorders in children. Research in Autism Spectrum Disorders, 1(1), 38-54.

Matson, J. L., Wilkins, J., \& Fodstad, J. C. (2011). the validity of the baby and infant screen for children with autism traits: Part 1 (BISCUIT: Part 1).
Journal of Autism and Developmental Disorders. 41(9), 1139-1146.

Matson, J. L., Wilkins, J., \& Gonzalez, M. (2008). Early identification and diagnosis in autism spectrum disorders in young children and infants: How early is too early? Research in Autism Spectrum Disorders, 2, $75-84$.

Ministério da Saúde. (2014). Diretrizes de atenção à reabilitação da pessoa com transtornos do espectro do autismo (TEA). Brasília, DF, Brasil.

Mitchell, S., Cardy, J. O., \& Zwaigenbaum, L. (2011). Differentiating autism spectrum disorder from other developmental delays in the first two years of life. Developmental Disabilities Research Reviews, 17(2), 130-140.

Nah, Y., Young, R. L., \& Brewer, N. (2014). Using the autism detection in early childhood (ADEC) and childhood autism rating scales (CARS) to predict long term outcomes in children with autism spectrum disorders. Journal of Autism and Developmental Disorders, 44(9), 2301-2310.

Nah, Y., Young, R. L., Brewer, N., \& Berlingeri, G. (2014). Autism detection in early childhood (ADEC): Reliability and validity data for a level 2 screening tool for autistic disorder. Psychological Assessment, 26(1), 215-226.

Nygren, G., Sandberg, E., Gillstedt, F., Ekeroth, G., Arvidsson, T., \& Gillberg, C. (2012). A new screening programme for autism in a general population of Swedish toddlers. Research in Developmental Disabilities, 33(4), 1200-1210.

Oosterling, I. J., Swinkels, S. H., van der Gaag, R. J., Visser, J. C., Dietz, C., \& Buitelaar, J. K. (2009). Comparative analysis of three screening instruments for autism spectrum disorder in toddlers at high risk. Journal of Autism and Developmental Disorders, 39(6), 897-909.

Organização Mundial da Saúde. (1993). Classificaşão Estatística Internacional de Doenças e Problemas Relacionados à Saúde - CID-10.

Pandey, J., Verbalis, A., Robins, D. L., Boorstein, H., Klin, A. M., Babitz, T., Chawarska, K., Volkmar, F., Green, J., Barton, M., \& Fein, D. (2008). Screening for autism in older and younger toddlers with the Modified Checklist for Autism in Toddlers. Autism, 12(5), 513-535. 
Papalia, D. E., Olds, S. W., \& Feldman, R.T. (2010). Desenvolvimento Humano. Tradução: VERCESI et al. 10. ed. Porto Alegre: AMGH.

Perera, H., Wijewardena, K., \& Aluthwelage, R. (2009). Screening of 18-24-month-old children for autism in a semi-urban community in Sri Lanka. Journal of Tropical Pediatrics, 55(6), 402-405.

Pool, J. L., \& Hourcade, J. J. (2011). Developmental screening: A review of contemporary practice. Education and Training in Autism and Developmental Disabilities, 46(2), 267-275.

Reichow, B., Barton, E. E., Boyd, B. A., \& Hume, K. (2012). Early intensive behavioral intervention (EIBI) for young children with autism spectrum disorders (ASD). Cochrane Database Syst Rev.

Reznick, J. S., Baranek, G. T., Reavis, S., Watson, L. R., \& Crais, E. R. (2007). A parent-report instrument for identifying one-year-olds at risk for an eventual diagnosis of autism: the first year inventory. Journal of Autism and Developmental Disorders, 37(9), 1691-1710.

Robins, D. L. (2008). Screening for autism spectrum disorders in primary care settings. Autism, 12(5), 537-556.

Robins, D. L., Casagrande, K., Barton, M., Chen, C. M. A., Dumont-Mathieu, T., \& Fein, D. (2014). Validation of the modified checklist for autism in toddlers, revised with follow-Up. Pediatrics, 133(1), $37-45$.

Rogers, S. J., Ozonoff, S., \& Hansen, R. L. (2013). Autism spectrum disorders. Em R. L., Hansen, S. J. Rogers (Eds.), Autism and other neurodevelopmental disorders (pp. 1-28) American Psychiatric Publishing.

Rogers, S. J., \& Vismara, L. (2014). Interventions for Infants and Toddlers at Risk for Autism Spectrum Disorder. Em F. R., Volkmar, S. J., Rogers, R., Paul, \& K. A. Pelphrey, (Eds.), Handbook of Autism Pervasive Developmental Disorders: assessment, interventions, and policy (4a ed.,Vol.2, pp. 739-769). Hoboken, New Jersey/EUA: Wiley.

Roux, A. M., Herrera, P., Wold, C. M., Dunkle, M. C., Glascoe, F. P., \& Shattuck P. T. (2012). Developmental and Autism screening through 2-1-1: reaching underserved families. American Journal of Preventive Medicine, 43(6 Supl. 5), 457-463.
Rutter, M. L. (2011). Progress in understanding autism: 2007-2010. Journal of Autism and Developmental Disorders, 41(4), 395-404.

Scambler, D. J, Hepburn, S. L., \& Rogers, S. J. (2006). A two-year follow-up on risk status identified by the checklist for autism in toddlers. Journal of Developmental and Behaviorial Pediatrics, 27(Supl. 2), 104-110.

Shattuck, P. T., Durkin, M., Maenner, M., Newschaffer, C., Mandell, D. S., Wiggins, L., Lee, L. C., Rice, C., Giarelli, E., Kirby, R., Baio, J., Pinto-Martin, J., \& Cuniff, C., (2009). Timing of identification among children with an autism spectrum disorder: finding from a population-based surveillance study. Journal of the American Academy of Child and Adolescent Psychiatry, 48, 474-483.

Stenberg, N., Bresnahan, M., Gunnes, N., Hirtz, D., Hornig, M., Lie, K. K., Lipkin, W. I., Lord., C., Magnus, P., Reichborn-Kjennerud, T., Schjølberg, S., Surén, P., Susser, E., Svendsen, B. K., von Tetzchner, S., Oyen, A. S., \& Stoltenberg C. (2014). Identifying children with Autism Spectrum Disorder at 18 months in a general population sample. Paediatric and Perinatal Epidemiology., 28(3), 255-262.

Stone, W. L., Coonrod, E. E., Turner, L. M., \& Pozdol, S. L. (2004). Psychometric properties of the STAT for early autism screening. Journal of Autism and Developmental Disorers, 6, 691-701.

Stone, W. L., McMahon, C. R., \& Henderson, L. M. (2008). Use of the Screening Tool for Autism in Two-Year-Olds (STAT) for children under 24 months. Autism, 12(5), 557-573.

Swinkels, S. H. N., Dietz, C., van Dallen, E., Kerkhof, I. H., van Engeland, H., \& Buitelaar, J. K. (2006). Screening for Autistic Spectrum in Children Aged 14 to 15 Months. I: The Development of the Early Screening of Autistic Traits Questionnaire (ESAT). Journal of Autism and Developmental Disorders, 36(6), 723-732.

Turner-Brown, L. M., Baranek, G. T., Reznick, J. S., Watson, L. R., \& Crais, E. R. (2012). The first year inventory: A longitudinal follow-up of 12-month -old to 3-year-old children. Autism, 0(0), 1-14.

Tsai, W. C., Soong, W.T., \& Shyu, Yi. (2012). Toddler autism screening questionnaire: Development and potential clinical validity. Autism, 16(4), 340-349. 
VanDenHeuvel, A., Fitzgerald, M., Greiner, B., \& Perry, I. J. (2007). Screening for autistic spectrum disorder at the 18-month developmental assessment: a population-based study. Irish Medical Joutnal, 100(8), 565-567.

Virués-Ortega, J. (2010). Applied behavior analytic intervention for autism in early childhood: Meta-analysis, meta-regression and dose-response meta-analysis of multiple outcomes. Clinical Psychology Review, 30(4), 387-399.

Wiggins, L. D., Piazza, V., \& Robins, D. L. (2014). Comparision of a broad-based screen versus disorder-specific screen in detecting young children with an autism spectrum disorder. Autism, 18(2), 76-84.
Wong, V., Hui, L. H. S., Lee, W. C., Leung L. S., Ho, P. K., Lau, W. L., Fung, C. W., \& Chung, B. (2004). A modified screening tool for autism (Checklist for Autism in Toddlers [CHAT-23]) for Chinese Children. Pediatrics, 114(2), 166-176.

Woolfenden, S., Sarkozy, V., Ridley, G., \& Williams, K. (2012). A systematic review of the diagnostic stability of Autism Spectrum Disorder. Research in Autism Spectrum Disorders, 6, 345-354.

Recebido em: 20/10/2015

$1^{\text {a }}$ Reformulação em: 16/03/2016

$2^{a}$ Refomulação em: 10/04/2016

Aceito em: 11/04/2016

Sobre as autoras:

Mariana de Miranda Seize é Psicóloga, Mestre e doutoranda em Psicologia Clínica pela Pontifícia Universidade Católica do Rio de Janeiro (PUC-Rio). Responsável pelo Grupo de Estudos sobre o Transtorno do Espectro Autista vinculado ao Grupo de Pesquisa em Avaliação Psicológica APlab - Pessoas \& Contextos. Certificada na formação clínica dos instrumentos internacionais ADI-R e ADOS-2 para diagnóstico do Transtorno do Espectro Autista.

E-mail: mariana.seize@gmail.com

Juliane Callegaro Borsa é Professora Adjunta do Departamento de Psicologia e do Programa de Pós-Graduação em Psicologia Clínica da Pontifícia Universidade Católica do Rio de Janeiro (PUC-Rio). Fundadora e coordenadora do Grupo de Pesquisa em Avaliação Psicológica APlab - Pessoas \& Contextos. Coordenadora do Laboratório de Avaliação Psicológica de Crianças e Adolescentes (LAPcriad). Supervisora de estágio profissionalizante no Serviço de Psicologia Aplicada (SPA/PUC-Rio).

E-mail: juliborsa@gmail.com

\section{Contato com as autoras:}

Pontifícia Universidade Católica do Rio de Janeiro

Rua Marquês de São Vicente 225, Edifício Cardeal Leme, $2^{\circ}$ Andar - Sala 201, Gávea

Rio de Janeiro-RJ, Brasil

CEP: 22451-900 